\title{
Temporally disordered Ising models
}

\author{
Juan José Alonso ${ }^{1}$ and Miguel A. Muñoz ${ }^{2}$ \\ 1 Departamento de Física Aplicada 1, Facultad de Ciencias, 29071 Málaga, Spain \\ ${ }^{2}$ Institute Carlos I for Theoretical and Computational Physics \\ and Departamento de E. y Física de la Materia, Universidad de Granada 18071 Granada, Spain.
}

(November 27, 2018)

\begin{abstract}
We present a study of the influence of different types of disorder on systems in the Ising universality class by employing both a dynamical field theory approach and extensive Monte Carlo simulations. We reproduce some well known results for the case of quenched disorder (random temperature and random field), and analyze the effect of four different types of time-dependent disorder scarcely studied so far in the literature. Some of them are of obvious experimental and theoretical relevance (as for example, globally fluctuating temperatures or random fields). All the predictions coming from our field theoretical analysis are fully confirmed by extensive simulations in two and three dimensions, and novel qualitatively different, non-Ising transitions are reported. Possible experimental setups designed to explore the described phenomenologies are also briefly discussed.
\end{abstract}

PACS numbers: 05.10 Cc, 05.10 Ln, 05.70 Ph, 64.60 Cn, 75.10 HK

The Ising model has played a central role in the development of modern statistical mechanics. Its universality class includes a broad variety of models and real systems, as magnetic and reaction-diffusion systems, spin glasses, neural networks, coupled maps, and protein folding models [1.2]. The analysis of the robustness of this universality class upon the introduction of disorder is of outmost importance in many different contexts. In fact, there are plenty of studies devoted to determining whether, and in which way, quenched disorder is a relevant perturbation at the Ising renormalization group (RG) fixed point 3 . 5. While a lot of attention has been paid to the study of quenched-in-space disorder [4, 30 in its different variants, quenched-in-time disorder (i.e. homogeneous disorder changing randomly in time) has not yet been thoroughly studied (some exceptions to this assertion are [6.7]; see also [2]). This is surprising as stochastically time-changing temperatures, or magnetic fields, are quite straightforward idealizations of realistic situations.

Here we study the effect of different types of disorder on the Ising universality class by using field theoretical tools and extensive Monte Carlo simulations. Quenchedin-space disorder will be considered for the sake of completeness (in particular, quenched-in-space temperature (QST), and quenched-in-space random field (QSF)), but we will chiefly focus on the novel and rich phenomenology of temporally disordered Ising models, and will analyze extensively the following four types of disorder: (1) Quenched-in-time, uniform-in-space, random temperature (QTT). (2) Quenched-in-space-time random temperature (QSTT). (3) Quenched-in-time, uniform-inspace, random (magnetic) field (QTF). (4) Quenched-inspace-time random (magnetic) field (QSTF). Physically, the difference between the odd and the even cases stems from the fact that in the first ones the fluctuating external parameter couples globally to the system, while even cases represent situations in which the fluctuating pa- rameter is inhomogeneous (changing in space and time). In terms on Monte Carlo implementations, this difference can be enviewed as associated with two different types of updating: if a value of the random variable is selected for each spin update (sequential updating), one is placed naturally at the second (or fourth) case, while if the random variable is extracted after updating of the whole lattice (this is the case, for example for parallel updating) the situation is described by the first (or third). In this way the type of updating can indirectly determine the nature of the disorder, and eventually the emerging behavior.

Our main conclusions are (listed in table 1): (i) QTT is a relevant perturbation for $d>2$, but irrelevant in $d=2$. We find strong numerical evidence of continuously varying exponents as a function of the width of the temperature distribution. A qualitatively different phenomenology is found for strong disorder (i.e. for widths above a certain threshold). (ii) QSTT is always an irrelevant perturbation. (iii) QTF is relevant for $d>1$; and a phenomenology completely different from the standard Ising one emerges. (iv) QSTF is marginal for $d=4$, and irrelevant otherwise.

Let us start discussing the disordered-temperature cases. The most basic result for QST disorder is the Harris criterion [8] that can be sketched as follows: If $T$ is a random uncorrelated [9] quenched-in-space variable, the amplitude of its fluctuations per unit volume, $f$, in a region of linear size $\xi$ is proportional to $\xi^{-d / 2}$. At a distance $\Delta$ of the critical point and taking $\xi$ to be the correlation length, one has $\xi \sim \Delta^{-\nu}$, and $f \propto \Delta^{\frac{\nu d}{2}}$. If $\nu d \leq 2$ temperature fluctuations control the critical scaling, while they are irrelevant otherwise [10]. To study the QTT disorder, one may replace $\xi$ by a characteristic time $t_{0} \sim \Delta^{\nu z}$ and follow analogous steps to obtain $\nu z \leq 2$ as the proper relevance criterion.

In order to make more rigorous and general the previous arguments let us now re-derive them in a field the- 
oretical context [8]. First we cast the Ising model into a Ginzburg-Landau-Wilson (GLW) dynamical functional [11], whose associated action is

$$
\begin{aligned}
\mathcal{S}= & \int d \mathbf{x} d t\left\{\frac{\lambda}{2} \psi^{2}(\mathbf{x}, t)-\psi(\mathbf{x}, t)[\dot{\phi}(\mathbf{x}, t)-\tau \phi-\right. \\
& \left.\left.\frac{\lambda}{2} \nabla^{2} \phi(\mathbf{x}, t)+\frac{g}{6} \phi^{3}(\mathbf{x}, t)+h(\mathbf{x}, t)\right]\right\}
\end{aligned}
$$

where $\phi$ is the magnetization field, $\psi$ is the response function, $\lambda$ and $g$ are parameters, $\tau \propto T-T_{c}$ includes the relevant dependence on the temperature [12], and $h$ is a magnetic field.

In order to include the effect of a disordered temperature we substitute $\tau$ by a function $\tau(\mathbf{x}, t)$. Then we can resort to one among two alternatives: one is to employ the "replica" trick [4, while here we follow the equivalent dynamical approach introduced some time ago by De Dominicis 13. In fact, the dynamical approach is the most natural one for the study of time-dependent disorder. The next step is to average the dynamical generating functional associated with Eq. (1) over the distribution of disorder. In particular, writing $\tau(\mathbf{x}, t)=\tau_{0}+\delta \tau(\mathbf{x}, t)$ (where $\tau_{0}$ is the averaged temperature and $\delta \tau(\mathbf{x}, t)$ is a zero-mean, $\sigma$ variance, Gaussian distributed random variable [9]) and performing the Gaussian integration, one obtains an effective action identical to Eq.(11) except for the replacement $\tau \rightarrow \tau_{0}$ and for the presence of the following additional disorder-dependent (nonlocal) terms:

$$
\begin{aligned}
& \sigma \int d \mathbf{x}\left[\int d t \phi(\mathbf{x}, t) \psi(\mathbf{x}, t)\right]^{2} \text { for } \quad Q S T \\
& \sigma \int d t\left[\int d \mathbf{x} \phi(\mathbf{x}, t) \psi(\mathbf{x}, t)\right]^{2} \text { for } Q T T \\
& \sigma \int d \mathbf{x} \int d t[\phi(\mathbf{x}, t) \psi(\mathbf{x}, t)]^{2} \text { for } \quad Q S T T \text {. }
\end{aligned}
$$

Let us analyze separately these three cases. For QST the naive dimension of the coefficient of the extra (nonMarkovian) term is such that Eq. (2) is dimensionless. As $\int d t \int d \mathbf{x} \tau_{0} \phi \psi$ has also to be dimensionless, $\left[\int d t \phi \psi\right]=\Lambda^{d-1 / \nu}$. Substituting this into the previous expression one obtains $[\sigma]=\Lambda^{2 / \nu-d}$, and therefore $\sigma$ is relevant (in the infrared limit [12]) if $d \nu \leq 2$ (reproducing the Harris criterion). Proceeding analogously for QTT one obtains $\nu z \leq 2$ and for QSTT $\nu(d+z) \leq 2$ as relevance criteria. Substituting the known Ising exponent values 12], one finds that: (see table) QST is a marginal perturbation at $d=2 \sqrt[14]{14}$ and relevant at $d=3$, as already known A, 16. QTT is relevant for $d \geq 3$, while it is irrelevant in $d=2$. QSTT is irrelevant in any dimension. This prediction implies that homogeneous globally changing temperatures (QTT) are far more effective in altering Ising behavior than locally changing temperatures (QSTT).

For the case of random fields, we proceed along analogous lines. By averaging the generating functional over a magnetic-field Gaussian distribution (centered at zero and of width $\delta$ ) [9], we obtain the terms to be added to Eq. (11): (i) $\delta \int d \mathbf{x}\left[\int d t \psi(\mathbf{x}, t)\right]^{2}$ for QSF (ii) $\delta \int d t\left[\int d \mathbf{x} \psi(\mathbf{x}, t)\right]^{2}$ for QTF, and (iii) $\delta \int d \mathbf{x} \int d t \psi^{2}(\mathbf{x}, t)$ and for QSTF.

By noticing that the dimension of $\int d t \psi(\mathbf{x}, t)$ at the pure Ising RG fixed point in terms of $\Lambda$ is $\frac{d}{2}-\frac{\gamma}{2 \nu}$ (where $\gamma$ is the susceptibility exponent), it is easy to derive the following relevance criteria: $\gamma / \nu \geq 0$ for QSF, $d \nu+\gamma \geq \nu z$ for QTF, and $\gamma \geq \nu z$ for QSTF. Substituting the Ising exponents [12 we are lead to the following predictions (see table 1): (i) QSF is relevant for $d>1$. For this case, the Imry-Ma argument establishes that $d=2$ is the lower critical dimension of the QSF universality class [16]). (ii) QTF is relevant in all dimensions. Some previous results about this model can be found [6]7; in particular, it is expected to exhibit a transition even in $d=1$, and a non Ising-like phase transition has been reported in $d=2$ [7]. (iii) QSTF is marginal only at $d=4$, and irrelevant otherwise (in fact, it just changes the noise amplitude, therefore it does not introduce any new operator). Analogously to what happens in the case of disordered temperatures, homogeneous global magnetic fields (QTF) are more effective in affecting the universal properties of the pure Ising class than magnetic fields varying both in time and in space (QSTF).

Let us finally stress that all the relevance criteria are determined using anomalous exponents, therefore at the Ising and not at the Gaussian fixed point. By performing naive power counting one can easily see, that $Q T T$ and $Q T F$ are also relevant perturbations at the Gaussian, mean-field, fixed point. Hence they are relevant even above the Ising upper critical dimension, $d_{c}=4$.

Having established all these relevance criteria, the next natural step would be to determine the new upper critical dimensions, $d_{c}$, in the cases where disorder is relevant, and perform an $\epsilon$-expansion in each case around the corresponding $d_{c}$. For example, for QSF the extra nonlocal operator scales as $\Lambda^{2}$ at $d=4$ (it is more relevant than the Ising nonlinearities), and accordingly the upper critical dimension is shifted from 4 to 6 . To every order in an $\epsilon$-expansion the exponents of the QSF model in $d$ dimensions are identical to those of the pure problem in $d-2$ dimensions 17.4.

Our attempts to perform similar detailed RG calculations in the other cases have turned out to be extremely hard and, so far, inconclusive [18]. For instance, for QTT and QTF the degree of divergence of the nonlocal operator is insensitive to dimensionality above the Ising critical dimension $d_{c}=4$. Consequently there is no upper critical dimension for these types of disorder which are strongly relevant even at high dimensions. Hence, even finding a sound mean-field approach, around which one could perform some sort of perturbative approach, turns out to be a nontrivial task. The only remaining possibility we can think of would be to perform a double expansion analogous to that in 19]. In any case, at the present stage 
of our research, we can just predict whether disorder is relevant or not, but cannot make sensible predictions concerning eventual new RG fixed points and the nature of new transitions.

In order to test the above predictions, and explore the eventual existence of new universality classes and/or novel phenomenologies, we have performed extensive Monte Carlo simulations combined with finite size scaling analysis in $d=2$ and $d=3$ with different types of disorder. We use: system sizes up to $L=512(L=90)$ in $d=2(d=3)$, periodic boundary conditions, Metropolis [2] transition rates, and run up to $3 \times 10^{7}$ Monte Carlo steps. Here we report the main results, further details and discussions will be presented elsewhere [20]:

(i) For disordered temperature cases we have performed simulations using both QTT and QSTT. The temperature is distributed homogeneously and symmetrically around a central (tunable) value $T_{0}$ and width $\delta$ [9]. For both types of disorder in $d=2$ we obtain exponents compatible with the respective systems being in the Ising class; see inset in Figure 1, where we study the scaling of the susceptibility peak (maximum value of the magnetization fluctuations) with system size $L$. The model studied in [21] can also be classified as QSTT in $d=2$, and in fact, it was reported to exhibit Ising scaling. In $d=3$ for QSTT from the curve $\chi$ vs $L$ we observe very good scaling using the three-dimensional $\gamma / \nu$ Ising value. On the other hand, for QTT we observe exponents varying continously with $\delta$, changing from the Ising value $\gamma / \nu=1.97(1)$ for $\delta=0$, to $\gamma / \nu=3$ for values of $\delta \approx 1.5$. There is no evidence of crossovers and the variation in exponent values is rather broad. Therefore we believe that these are the real asymptotic values and that accordingly there should be a line of RG fixed points. Caution should be used before concluding that the exponents actually change continuously. Corrections to scaling could be responsible for such a variation [22]. For values of $\delta$ larger than a certain threshold around 1.5 the exponent $\gamma / \nu$ saturates to 3 implying that the magnetization histograms do not converge to delta Dirac functions in the thermodynamic limit: they remain broad, corresponding to external-fluctuations-controlled transitions.

(ii) For the case of random fields, QTF is expected to be non-Ising in $d=2$, while QSTF disorder is predicted to be an irrelevant perturbation. In fact, for QSTF our simulations show a perfect agreement with Ising exponents for all the measured magnitudes; non-universal properties depend on the disorder width, $\delta$, but critical exponents remain unchanged. The models studied in 23.2] can also be ascribed to this class of disorder, and were found to be Ising like in $d=2$ in accordance with our prediction. On the other hand for QTF we observe a completely different phenomenology: There is a continuous phase transition whose features are unambiguously different from the corresponding Ising ones. In particular, it is a sort of dynamical transition controlled by the fluctuations of the randomly changing magnetic field. For example, in figure 2, we show the dependence of the susceptibility per unit surface as a function of system size, for different values of $\delta$. After a finite-size-effect transient, the curves converge to a fixed value, indicating the existence of broad magnetization histograms in the thermodynamic limit, and implying $\gamma / \nu=2$. Still, magnitudes as the specific heat or the susceptibility show singular peaks at the critical point, and there is a dynamical spontaneous symmetry breaking below the critical temperature 20,24. We have reproduced qualitatively this QTF phenomenology by means of a dynamical mean-field approach to be reported elsewhere [20], and observed that the nature of this transition does not seem to be very sensible to dimensionality changes.

Summing up: we have presented a rather simple general field theoretical scheme allowing us to predict the relevance of different types of disorder in the Ising universality class. Some of the conclusions where already known, and provide a validity test for our approach. Using this scheme we have studied the effect of different types of time-dependent disorder in the Ising class. These disordered models describe rather natural situations in which systems are subjected to the influence of some globally (or locally) fluctuating external agent (randomly varying temperatures or magnetic fields). All our predictions are fully confirmed by means of extensive Monte Carlo simulations; and novel quite interesting phenomenologies appear for the case of homogeneously fluctuating temperatures and magnetic fields.

In order to experimentally verify our predictions one could take, for example, vacuum deposited ultrathin Fe films on W(110) (i.e. a nearly ideal two-dimensional magnetic Ising system 25]), and in the same way that this material has been set to study hysteresis in the presence of an oscillating magnetic field 25, it can be analyzed under the effect of a randomly varying magnetic field (generated, for example, by means of an electric circuit in a chaotic regime). A detailed comparison between the characteristic relaxation time-scale, and the typical magnetic field variation scale is of outmost importance in order to determine the applicability of our results to this type of experiments.

$A C K N O W L E D G M E N T S$ - It is a pleasure to acknowledge J. Marro, P. L. Garrido, and J.J. Ruiz-Lorenzo for useful discussions and criticisms. This work has been partially supported by the European Network Contract ERBFMRXCT980183 and by the Ministerio de Educación: projects DGESEIC PB97-0842 and PB97-1080.

[1] H. E. Stanley, Introduction to Phase Transitions and Critical Phenomena, Oxford science, 1987.

[2] J. Marro and R. Dickman, Nonequilibrium Phase Transitions in Lattice Models, Cambridge University Press, (Cambridge, 1999). 
[3] R. B. Stinchcombe, Dilute Magnetism, in Phase Transitions and critical phenomena, vol 7. eds. C. Domb and J. L. Lebowitz. Academic Press. London (1983).

[4] See G. Grinstein in Fundamental Problems in Statistical Mechanics VI, Edited by E. G. D. Cohen, Elsevier Science, 1985, and references therein.

[5] J. L. Cardy, Scaling and Renormalization in Statistical Physics, Cambridge Univ. Press, 1997.

[6] M. Acharyya, Phys. Rev. E 58, 174 (1998).

[7] See J. Hausmann and P. Ruján, Phys. Rev. Lett. 79, 3339 (1997); J. Phys. A bf 32, 61 (1999); ibid bf 32, 75 (1999); and references therein.

[8] A. B. Harris, J. Phys. C 7, 1671 (1974). The criterion is supported by several RG analysis [- 4 , and has been extended to strong disorder cases by J. T. Chayes, et al. Phys. Rev. Lett. 57, 2999 (1986).

[9] As long as the disorder at different sites is uncorrelated, details of the one-site distribution are irrelevant.

[10] Using the scaling law $\nu d=2-\alpha$ [12] the relevance criterion can be written in its more familiar form, $\alpha>0$.

[11] R. Bausch et al., Z. Phys. B 24, 113, (1976).

[12] D. Amit, Field Theory, the Renormalization group and critical phenomena, World Scientific, 1984. G. Parisi, Statistical Field Theory, Addison Wesley, 1988.

[13] C. De Dominicis, Phys. Rev. B18, 4913, (1978).

[14] Recent results concerning the 2D QST model can be found in S. L. A. Queiroz, Comp. Phys. Comm. 121122, 210 (1999); and references therein.

[15] Y. Imry and S. -K. Ma, Phys. Rev. Lett. 35, 1399 (1975).

[16] Whether QSF in $d=3$ defines a unique universality class or there are disorder-distribution dependent RG fixed points is a presently debated issue. See N. Sourlas, in Comp. Phys. Comm. 121-122, 183 (1999); H. G. Ballesteros et al. Phys. Rev. B 58, 2740 (1998).

[17] This dimensional reduction [G. Parisi and N. Sourlas, Phys. Rev. Lett. 43, 744 (1979) ] is at odds with the Imry-Ma prediction [15], and it is only recently that this puzzle has been elucidated: See E. Brezin and C. De Dominicis, Europhys. Lett. 44, 13 (1998).

[18] A similar renormalization attempt for disordered systems in the directed percolation class can be found in H. K. Janssen, Phys. Rev. E 55, 6253 (1997).

[19] J. Cardy and U. Taeuber, Phys. Rev. Lett. 77, 4870 (1996).

[20] J. J. Alonso and M. A. Muñoz, preprint 2000.

[21] J. Marro et al. Phys. Rev. E 50, 3237 (1994).

[22] The existence of continuously changing exponents in: (1) systems in the directed percolation class with this type of disorder (proved numerically and using series expansions in I. Jensen, Phys. Rev. Lett. 77, 4988 (1996)) and, (2) a one dimensional model with the same type of disorder [7], supports our belief.

[23] J. J. Alonso, A.I. López and J. Marro, Phys. Rev. E 52, 6006 (1995).

[24] A similar situation was studied in $\mid 7$ : the disorder distribution was bimodal there, and this made the transition to be a first-order one.

[25] H. J. Elmers, Int. J. Mod. Phys. B 9, 3115 (1995). J. S. Suen and J. L. Erskine, Phys. Rev. Lett. 78, 3567 (1997).

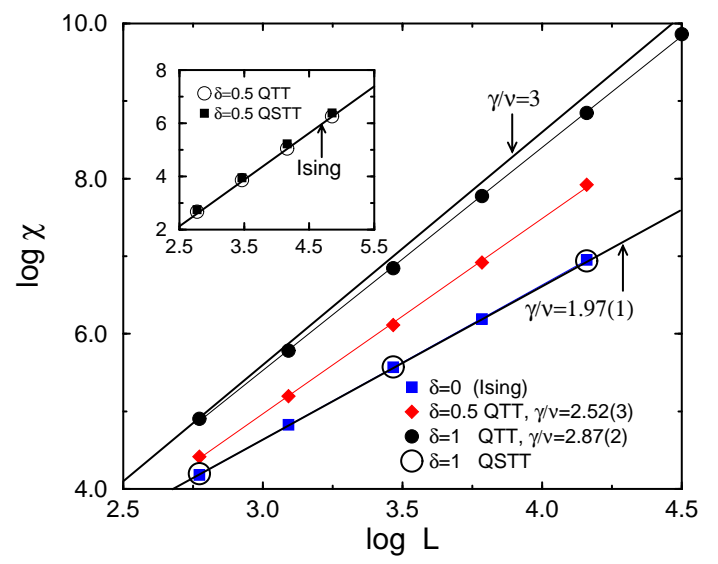

FIG. 1. Susceptibility at the critical point as a function of system size for disordered-temperature models in $d=3$ $(d=2$ in the inset). QSTT exhibits Ising scaling in $d=3$. For QTT the slopes (fixing $\gamma / \nu$ ) change continuously with disorder amplitude in $d=3$, but not in $d=2$ (see inset).

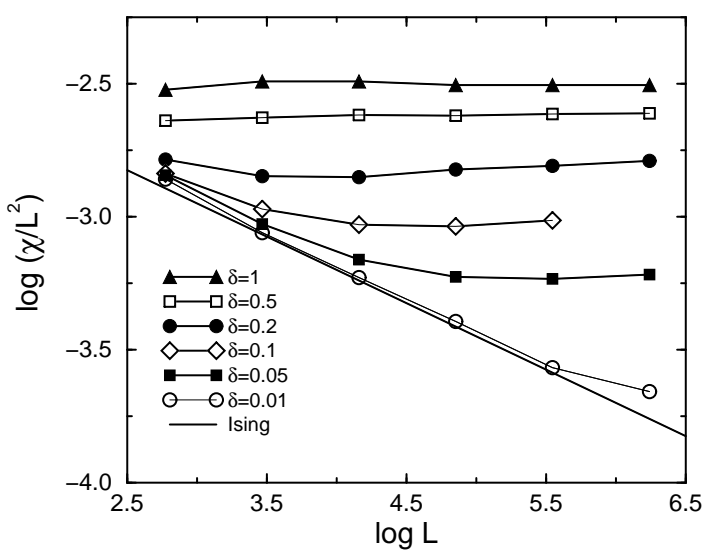

FIG. 2. Susceptibility per unit surface as a function of $\mathrm{L}$ (in log-log scale) for QTF disorder. For large sizes the curves converge to a constant indicating the existence of broad magnetization histograms in the thermodynamic limit.

\begin{tabular}{|c|c|c|c|}
\hline \hline DISORDER & Space & Time & Space-Time \\
\hline \hline & $Q S T$ & $Q T T$ & $Q S T T$ \\
Random & $d \nu \leq 2$ & $\nu z \leq 2$ & $\nu(d+z) \leq 2$ \\
Temperature & $d \geq 2$ & $d \geq 3$ & --- \\
\hline & $Q S F$ & $Q T F$ & $Q S T F$ \\
Random & $\gamma / \nu \geq 0$ & $d \nu+\gamma \geq \nu z$ & $\gamma>\nu z$ \\
Field & $d>1$ & $d \geq 1$ & $d=4$ marginal \\
\hline \hline
\end{tabular}

TABLE I. Criteria for the relevance of different types of disorder in the Ising universality class. The first row in each case specifies the general criteria (equalities correspond to marginal cases), while in the second we specify the dimensions at which such criteria are fulfilled. 